\title{
Pimecrolimus in dermatology: atopic dermatitis and beyond
}

\author{
PAOLO GISONDI, ${ }^{1}$ CHARLES N. ELLIS, ${ }^{2}$ GIAMPIERO GIROLOMONI ${ }^{3}$ \\ Department of Dermatology, ${ }^{1}$ Istituto Dermopatico dell'Immacolata, IRCCS, Rome, Italy, Department of Dermatology, ${ }^{2}$ University \\ of Michigan Medical School, Ann Arbor, MI, USA, Section of Dermatology, Department of Biomedical and Surgical \\ Sciences, ${ }^{3}$ University of Verona, Verona, Italy
}

\section{SUMMARY}

Pimecrolimus is a calcineurin inhibitor developed for the topical therapy of inflammatory skin diseases, particularly atopic dermatitis (AD). Pimecrolimus selectively targets $\mathrm{T}$ cells and mast cells. Pimecrolimus inhibits T-cell proliferation, as well as production and release of interleukin-2 (IL-2), IL-4, interferon $-\gamma$ and tumour necrosis factor- $\alpha$. Moreover, pimecrolimus inhibits mast cell degranulation. In contrast to tacrolimus, pimecrolimus has no effects on the differentiation, maturation and functions of dendritic cells. In contrast to corticosteroids, pimecrolimus does not affect endothelial cells and fibroblasts and does not induce skin atrophy. Given the low capacity of pimecrolimus to permeate through the skin, it has a very low risk of systemic exposure and subsequent systemic side-effects. In different randomised controlled trials, topical pimecrolimus as cream $1 \%$ (Elidel $^{\circledR}$ ) has been shown to be effective, well tolerated and safe in both adults and children with mild to moderate AD. In addition, pimecrolimus has been successfully used in inflammatory skin diseases other than $\mathrm{AD}$, including seborrheic dermatitis, intertriginous psoriasis, lichen planus and cutaneous lupus erythematosus.

Keywords: Pimecrolimus; inflammatory skin disease; therapy; atopic dermatitis

(C) 2005 Blackwell Publishing Ltd

\section{INTRODUCTION}

For many years, topical corticosteroids have been the mainstay of dermatological treatment for many inflammatory skin diseases. Nowadays, the topical calcineurin inhibitors, pimecrolimus and tacrolimus, provide an effective and safe alternative especially for long-term control of chronic inflammatory diseases such as atopic dermatitis (AD). This review focuses on the mechanism of the action of pimecrolimus, the evidence regarding the efficacy and safety of topical pimecrolimus in AD and its use in 'off-label' skin conditions.

\section{MECHANISM OF ACTION}

Pimecrolimus is a macrolactam immunomodulator belonging, together with tacrolimus and cyclosporine, to the family of calcineurin inhibitors. These drugs bind to cytoplasmic proteins and the resulting complex binds calcineurin, inhibiting its ability to dephosphorylate the nuclear factor of activated $\mathrm{T}$ cells (NF-AT). The ligand for cyclosporine is cyclophilin,

\section{Correspondence to:}

Giampiero Girolomoni, Section of Dermatology, Department of Biomedical and Surgical Sciences, University of Verona, Piazzale Stefani 1, 37126 Verona, Italy

Tel.: + 390458072547

Email: giampiero.girolomoni@univr.it whereas pimecrolimus and tacrolimus bind macrophilin-12, also known as FK506-binding protein.

NF-AT is a nuclear transcription factor that facilitates the transcription of several growth factor and inflammatory genes; however, it must be phosphorylated to translocate into the nucleus (1). Pimecrolimus shows a selective action on $\mathrm{T}$ cells and mast cells as opposed to the more pleiotropic targets of tacrolimus and corticosteroids (Table 1). The activation of $\mathrm{T}$ cells and mast cells plays a crucial role in the pathogenesis of many inflammatory skin diseases, including AD. Pimecrolimus inhibits $\mathrm{T}$-cell proliferation and production and the release of several growth factors and pro-inflammatory cytokines including interleukin-2 (IL-2), IL-4, interferon- $\gamma$ (IFN- $\gamma$ ) and tumour necrosis factor- $\alpha$ (TNF- $\alpha$ ). Moreover, pimecrolimus prevents mast cell release of pro-inflammatory mediators including histamine, cytokines, tryptase and eicosanoids $(2,3)$.

In contrast to tacrolimus and corticosteroids, pimecrolimus does not affect the differentiation, maturation and functions of dendritic cells and does not induce apoptosis of epidermal Langerhans' cells (4-6). In line with these findings, pimecrolimus administered to mice suppresses only the elicitation (but not the sensitisation) phase of contact hypersensitivity to haptens, a T-cell-mediated immune reaction driven by dendritic cells that represents a model of human allergic contact dermatitis (7). In contrast to corticosteroids, pimecrolimus and tacrolimus do not affect endothelial cells and fibroblasts and, therefore, do not induce telangiectasia and skin atrophy (8). 
Table 1 Comparison between topical pimecrolimus, tacrolimus and corticosteroids

\begin{tabular}{llll}
\hline & Pimecrolimus & Tacrolimus & Corticosteroids \\
\hline Major cellular targets & T cells, mast cells & $\begin{array}{l}\text { T cells, mast cells, } \\
\text { dendritic cells, eosinophils }\end{array}$ & $\begin{array}{l}\text { T cells, mast cells, dendritic cells, endothelial cells, } \\
\text { Cytokines inhibited }\end{array}$ \\
& IL-2, IL-3, IL-4, IL-5, & IL-2, IL-3, IL-4, IL-5, & IL-2, IL-3, IL-4, IL-5, GM-CSF, \\
& IFN- $\gamma$, TNF- $\alpha$ & IFN- $\gamma$, TNF- $\alpha$ & IFN- $\gamma$, TNF- $\alpha$, IFN- $\alpha$ \\
Blockade of dendritic cell functions & - & + & ++ \\
Apoptosis of dendritic cells & - & + & ++ \\
Suppression of the sensitisation & - & + & + \\
phase of contact hypersensitivity & & & + \\
$\begin{array}{l}\text { Suppression of the elicitation } \\
\text { phase of contact hypersensitivity }\end{array}$ & + & + & ++ \\
Skin penetration & + & ++ & +++ \\
Atrophogenic potential & - & - & + \\
\hline
\end{tabular}

Permeation of topical drugs through the skin leading to uptake into the systemic circulation is generally not desired and may, in some instances (e.g. in children, on extensive medication of large skin areas), lead to systemic side-effects. In the case of topical corticosteroids, such side-effects may include hypothalamic-pituitary-adrenal axis suppression and Cushing's syndrome. The propensity of pimecrolimus to pass through the skin is about 90 times lower than corticosteroids and about nine times lower than tacrolimus (9). The differences related to skin permeation may be explained by the distinct lipophilicity/hydrophilicity distribution within the molecules, and the higher molecular weight of pimecrolimus and tacrolimus (approximately $800 \mathrm{Da}$ ), compared with corticosteroids (approximately $470 \mathrm{Da}$ ). The intrinsic capability of pimecrolimus and tacrolimus to cross the stratum corneum is similar, whereas the further penetration is impaired in the case of pimecrolimus. We can thus assume that the percutaneous absorption of pimecrolimus into systemic circulation is not relevant clinically. Moreover, the more selective action of pimecrolimus may account for a lower risk of systemic immune suppression, thus offering a better safety margin than other topical drugs.

\section{PIMECROLIMUS IN ATOPIC DERMATITIS}

Atopic dermatitis is a chronic inflammatory skin disease affecting $5-20 \%$ of children and $1-3 \%$ of adults $(10,11)$. The majority of cases begins in childhood, often in infancy. $\mathrm{AD}$ runs a chronic, relapsing course and manifests as an itchy, erythematous and scaly rash commonly on the face and skin folds (neck, knees and elbows). AD is a heterogeneous disease frequently associated with asthma and rhino-conjunctivitis. AD has a significant impact on the quality of life of patients and caregivers, causes sleep disturbances and affects patients' social and school/work life. AD imposes a financial burden on the health care system and even more on the individual for both direct and indirect costs $(12,13)$
The pathogenesis of $\mathrm{AD}$ is complex with both genetic and environmental factors playing important roles. Both these components stimulate an excessive type 2 immune response to allergens and skin inflammatory reactivity. Cutaneous lesions may result from an immune response to allergens and also from an abnormal cutaneous reactivity to irritants, both favoured by epidermal barrier dysfunction (10).

The therapeutic approach to $\mathrm{AD}$ patients includes the identification and elimination of triggering environmental factors. The goal of treatment is to induce and maintain remission. The therapy includes appropriate skin care, topical glucocorticoids and topical calcineurin inhibitors. Pruritus is only partially controlled by systemic $\mathrm{H} 1$-antihistamines. Treatment with phototherapy and with systemic glucocorticoids and other immunosuppressives is restricted to patients with severe disease.

Pimecrolimus is currently approved by the Food and Drug Administration (FDA) and the European Medicines Agency for short-term and intermittent long-term treatment of mild to moderate $\mathrm{AD}$ in non-immunocompromised patients 2 years of age or older. Numerous double-blind, controlled clinical trials proved the efficacy and safety of pimecrolimus in $\mathrm{AD}$.

\section{Efficacy of Pimecrolimus in AD}

Short-term studies. In two independent 6-week randomised control trials (RCTs), treatment with pimecrolimus $1 \%$ cream twice daily alleviated significantly signs and symptoms of $\mathrm{AD}$ in children and adolescents as compared with its emollient vehicle. At week 6, 35\% of pimecrolimus-treated patients were classified as clear or almost clear of disease according to an Investigator Global Assessment (IGA). The drug showed a rapid onset of action, with therapeutic effects observed by the first visit on day 8 and further improvement thereafter (14). The ethnic origin and the baseline disease severity had no effect on treatment outcome (15). The effects 
of pimecrolimus compared with vehicle on sleep disturbances were also evaluated. Although not significant after 3 weeks of therapy, all trends suggested better sleep patterns in the group treated with pimecrolimus cream (16).

An RCT compared pimecrolimus cream 1\% with tacrolimus ointment $0.03 \%$ in paediatric patients with moderate to severe AD. A total of 170 patients aged 2-17 years were randomised into two treatment groups (pimecrolimus vs. tacrolimus). The therapies were applied twice daily until complete clearance of disease or until week 6. Regarding efficacy, there were no statistically significant differences between treatment groups in the proportion of patients achieving clinical success (i.e. IGA or pruritus score of 0 or 1); however, IGA response rates were slightly higher in the tacrolimus group. Application site reactions (ASRs) were significantly more common and of longer duration in the tacrolimus group than in the pimecrolimus group. The formulation attributes of pimecrolimus were generally preferred by patients or caregivers compared with tacrolimus. Local viral or bacterial skin infections were infrequent (involving no more than $3 \%$ of patients), and no major differences were observed between treatment groups. This trial showed that pimecrolimus cream $1 \%$ and tacrolimus ointment $0.03 \%$ have a similar efficacy in the treatment of paediatric patients with moderate to severe $\mathrm{AD}$, but the pimecrolimus formulation was generally preferred (17).

Long-term studies. In a 1-year, multicentre RCT involving 251 infants aged 3-23 months with AD, pimecrolimus cream $1 \%$ was compared with its vehicle in the long-term management of mild to moderate AD. Topical treatment with pimecrolimus significantly reduced the incidence of flares compared with vehicle, with 68 vs. $30 \%$ of patients completing 6 months with no flares and 57 vs. $35 \%$ completing 12 months with no flares. These data suggest that the use of pimecrolimus at the early signs and symptoms of AD significantly modifies disease course by reducing the occurrence of flares (18). This study was followed by an open-label extension of 1 year, in which the proportion of patients with no flares increased over time to $85 \%$ (19). The potential of pimecrolimus to prevent flares of $\mathrm{AD}$ requiring corticosteroid therapy was evaluated also in adults. A 6-month study in adults with moderate $\mathrm{AD}$ demonstrated that the early administration of pimecrolimus at the first signs of disease exacerbation prevented progression to flares and significantly reduced, compared with emollients, the need for topical corticosteroids in almost $60 \%$ of patients (20).

The long-term safety and efficacy of pimecrolimus in a large adult population $(n=658)$ were compared with topical corticosteroids $(0.1 \%$ triamcinolone acetonide for the trunk and limbs and $1 \%$ hydrocortisone acetate for the face, neck and intertriginous area). Medications were applied twice daily to all affected areas until complete clearance of the inflammation was achieved and pruritus had ceased. In efficacy measures, patients treated with corticosteroids responded better than pimecrolimus-treated patients at all time points except at the end of the study (after 13 months) when the differences were not statistically significant. An interesting distinction between the two groups was the proportion of patients that discontinued the study prematurely for unsatisfactory therapeutic effect, namely $36 \%$ of the patients receiving pimecrolimus and $8 \%$ of those on corticosteroid treatment. The decision of patients treated with pimecrolimus to discontinue the study appears to correlate with the severity of disease before starting therapy. Among patients receiving pimecrolimus, the risk of discontinuation for the lack of efficacy in patients with Eczema Area and Severity Index (EASI) score $>25$ at baseline was 2.6 times higher than the risk for patients with a baseline EASI score of 5-15, and the risk for patients with a baseline EASI score $<5$ was about half that of patients with a baseline EASI score of 5-15. Therefore, severity of disease is a predictor of premature discontinuation.

There were no statistically significant differences between the two treatment groups in the global incidence of bacterial, fungal and viral skin infections. However, in patients with $>30 \%$ of the body surface area affected by $\mathrm{AD}$, and thus requiring more extensive medication, the incidence of overall skin infections in the group receiving corticosteroids was double that in the pimecrolimus group (21).

\section{Safety of Pimecrolimus in AD}

After subcutaneous or oral administration in mice, pimecrolimus showed a lower potential of affecting immune responses compared with tacrolimus (22). Indeed, patients with AD treated with pimecrolimus cream $1 \%$ for up to 1 year showed a normal response pattern to a range of common bacterial and fungal antigens (23). Moreover, treatment of $\mathrm{AD}$ with pimecrolimus cream $1 \%$ in early childhood for 2 years did not interfere with the development of a normal immune response to vaccinations (24).

Local side-effects of topical calcineurin inhibitors commonly reported by patients are feelings of warmth/burning/ stinging, increase of erythema or irritation and increased itching. In a short-term study in paediatric patients, feeling of warmth/burning/stinging was reported by $20 \%$ of patients, whereas erythema/irritation and itching was reported by $8 \%$. All lasted less than $30 \mathrm{~min}$ (17). In a long-term study in infants, there were no clinically significant differences between the group receiving pimecrolimus and the group receiving placebo with respect to the incidence of common adverse events and skin infections (18).

In the long-term study conducted in adults, ASRs were experienced by $46 \%$ of patients. In general, these events were of mild to moderate severity in most cases and occurred early in the treatment phase. In $50-60 \%$ of cases, ASRs started 
during the first 4 days of pimecrolimus application, resolved within 7 days and were most frequently localised on the face and neck. The most common ASR was burning sensation, which occurred in $26 \%$ of the adult patients treated with pimecrolimus.

Comparing the incidence of infections in adult patients treated with pimecrolimus or vehicle, bacterial and fungal infections occurred at similar frequencies in both groups. However, viral infections, specifically herpes simplex infection, was more frequent in the pimecrolimus group (11\%) vs. vehicle (4\%) (20). No patient developed eczema herpeticum. Another critical concern is the potential risk of the development of ultraviolet radiation (UVR)-mediated skin cancer. The risk of photocarcinogenicity associated with pimecrolimus and exposure to sunlight was investigated in a standard murine model. After 40 weeks of daily UVR exposure and topical concomitant application of pimecrolimus, no increase in the incidence of precancerous lesions was observed in comparison with that in the vehicle-treated animals, giving evidence for a low photocarcinogenic property of pimecrolimus (FDA/Center for Drug Evaluation and Research. Elidel cream. Retrieved from http://www.fda.gov/cder/foi/nda/ 2001/21-302). No phototoxic or photoallergic potential was observed in healthy individuals.

Therapy with the calcineurin inhibitors and corticosteroids may, on a theoretical basis, increase the risk of development of non-cutaneous cancers, including lymphoma. There is no evidence to indicate that any such increase in risk occurs with topical therapy in humans, although significant systemic immunosuppression in animals and humans does increase cancer risk.

There are no conclusive data regarding the safety of pimecrolimus during pregnancy. Pimecrolimus is excreted in human milk; therefore, its use is not recommended during nursing.

\section{PIMECROLIMUS IN OTHER SKIN DISEASES}

Topical pimecrolimus may be useful in the treatment of chronic inflammatory skin diseases other than AD. The efficacy of pimecrolimus according to the level of evidence is presented in Table 2. Controlled trials have provided evidence of efficacy of topical pimecrolimus in intertriginous psoriasis (25), seborrheic dermatitis (26) and chronic hand dermatitis (27). In addition, pimecrolimus has been found to be effective in oral lichen planus $(28,29)$ and cutaneous lupus erythematosus (30). Case reports of clinical success have been reported for cutaneous lichen planus (31), vitiligo (32) and chronic graft-vs.-host disease (33).

\section{Psoriasis}

The efficacy of pimecrolimus in the therapy of inverse psoriasis has been documented in a randomised, double-blind, placebo-controlled clinical trial involving 57 adult patients. A large proportion (i.e. 71\%) of patients treated with pimecrolimus was assessed as clear or almost clear by the investigator after therapy for 8 weeks; however, the benefits of the therapy were observed as early as day 3. Only one case of mild application site paresthesia suspected to be related to the topical pimecrolimus was reported, whereas skin atrophy, telangiectasia and secondary bacterial or Candida skin infection were not reported during the study. In another randomised, double-blind study, the efficacy of an ointment formulation of pimecrolimus $1 \%$ was compared with its vehicle, with $0.005 \%$ calcipotriol and with $0.05 \%$ clobetasol17-propionate in the treatment of plaque psoriasis. At day 21, pimecrolimus ointment applied without occlusion was more effective than its vehicle, but significantly less effective than calcipotriol and clobetasol in reducing erythema, induration and scaling scores (34).

Table 2 Pimecrolimus in inflammatory skin diseases

\begin{tabular}{lll}
\hline Established efficacy & Likely beneficial & Possibly beneficial \\
\hline Atopic dermatitis $(1++)$ (mild to moderate) & Seborrheic dermatitis (2-) & Vitiligo (3) \\
Intertriginous psoriasis (1+) & Anogenital lichen sclerosus and atrophicus (3) & Lupus erythematosus (3) \\
& Oral and cutaneous lichen planus (3) & Dermatomyositis (3) \\
& Chronic graft-vs.-host disease (3) \\
\hline
\end{tabular}

Level of evidence according to the Scottish Intercollegiate Guidelines Network (37):

$(1++)$ High-quality meta-analyses, systematic reviews of randomised control trials (RCTs) or RCTs with a very low risk of bias.

(1+) Well-conducted meta-analyses, systematic reviews of RCTs or RCTs with a low risk of bias.

(1-) Meta-analyses, systematic reviews or RCTs or RCTs with a high risk of bias.

$(2++)$ High-quality systematic reviews of case-control or cohort studies or high-quality case-control or cohort studies with a very low risk of confounding, bias or chance and a high probability that the relationship is causal.

(2+) Well-conducted case-control or cohort studies with a low risk of confounding, bias or chance and a moderate probability that the relationship is causal.

(2-) Case-control or cohort studies with a high risk of confounding, bias or chance and a significant risk that the relationship is not causal.

(3) Non-analytic studies, e.g. case reports and case series.

(4) Expert opinion. 


\section{Seborrheic Dermatitis}

The efficacy of pimecrolimus in the treatment of seborrheic dermatitis has been compared with a potent corticosteroid in an open-label clinical trial involving 22 adult patients (26). The efficacy of the two different treatments in reducing erythema, scaling and pruritus was approximately the same. Both drugs reduced symptoms completely at day 9, with betametasone acting faster than pimecrolimus. However, following discontinuation of treatment, relapses were observed more frequently and were more severe in the betamethasone than in the pimecrolimus group.

\section{Chronic Hand Dermatitis}

In a large, randomised, double-blind, vehicle-controlled study involving 294 adults patients with chronic hand dermatitis, pimecrolimus induced a complete or almost complete clearance in $30 \%$ of patients after 22 days of continuous therapy including overnight occlusion (27). The proportion of patients achieving treatment success was greater in the active group compared with the placebo group, but the difference was not statistically significant. Patients without palmar involvement responded better to treatment than those without palmar involvement, perhaps because the thickness of the stratum corneum on the palms could impair penetration of the drug. The overnight occlusion in subjects with chronic hand dermatitis does not lead to a massive permeation through skin, because the pimecrolimus blood concentration was consistently low through the study and, in $74 \%$ of cases, below the limit of quantification $(0.1 \mathrm{ng} / \mathrm{ml})(35)$.

\section{Oral Pimecrolimus}

Testing the efficacy of oral pimecrolimus in patients with psoriasis or AD is underway. Data currently available show that pimecrolimus is effective in the therapy of chronic plaque psoriasis in a dose-dependent manner. At week 12, the median reduction of the Psoriasis Area and Severity Index (PASI) score was 80 and $58 \%$ in 60 and $40 \mathrm{mg} /$ day dosage groups, respectively. The drug showed a good safety profile in short-term treatment; the only consistent side-effect recorded was a transient feeling of warmth on the upper chest occurring $40 \mathrm{~min}$ after ingesting the medication and lasting about 90 min. Moreover, with short-term treatment, pimecrolimus had no significant effect on laboratory measures nor did it affect various immunologic parameters. Intradermal testing for delayed hypersensitivity reactions to recall antigens showed no significant changes after the course of pimecrolimus therapy (36). The long-term efficacy, time-to-relapse after discontinuation and the long-term safety await further studies.

\section{CONCLUSIONS}

Pimecrolimus cream appears an effective and safe treatment option for the treatment of $\mathrm{AD}$, especially when the disease is mild to moderate and affects the face and neck regions. Moreover, if the drug is applied at the first signs of exacerbation of $\mathrm{AD}$, it prevents flare progression, modifying the course of the disease and reducing the need for topical corticosteroids. In infants and children, a better control of $\mathrm{AD}$ may reduce the risk of subsequent development of asthma and/or rhinitis (atopic march). Finally, pimecrolimus may well work in other inflammatory skin diseases particularly when lesions are not keratotic and are localised on the face or skin folds (where corticosteroids are more likely to induce side-effects). Examples of such conditions include seborrheic dermatitis and intertriginous psoriasis.

\section{REFERENCES}

1 Marsland AM, Griffiths CE. The macrolide immunosuppressants in dermatology: mechanisms of action. Eur $J$ Dermatol 2002; 12: 618-22.

2 Grassberger M, Steinhoff M, Schneider D, Luger TA. Pimecrolimus - an anti-inflammatory drug targeting the skin. Exp Dermatol 2004; 13: 721-30.

3 Graham-Brown RAC, Grassberger M. Pimecrolimus: a review of pre-clinical and clinical data. Int J Clin Pract 2003; 57: 319-27.

4 Hoetzenecker W, Meingassner JG, Ecker R et al. Corticosteroids but not pimecrolimus affect viability, maturation and immune function of murine epidermal Langerhans cells. J Invest Dermatol 2004; 122: 673-84.

5 Kalthoff FS, Chung J, Musser P, Stuetz A. Pimecrolimus does not affect the differentiation, maturation and function of human monocyte-derived dendritic cells, in contrast to corticosteroids. Clin Exp Immunol 2003; 133: 350-9.

6 Panhans-Gross A, Novak N, Kraft S, Bieber T. Human epidermal Langherans' cells are targets for the immunosuppressive macrolide tacrolimus (FK506). J Allergy Clin Immunol 2001; 107: 345-52.

7 Meingassner JG, Fahrngruber H, Bavandi A. Pimecrolimus inhibits the elicitation phase but does not suppress the sensitization phase in murine contact hypersensitivity in contrast to tacrolimus and cyclosporine A. J Invest Dermatol 2003; 121: 231.

8 Queille-Roussel C, Paul C, Duteil L et al. The new topical ascomycin derivative SDZ ASM 981 does not induce skin atrophy when applied to normal skin for 4 weeks: a randomized, double-blind controlled study. Br J Dermatol 2001; 144: 507-13.

9 Billich A, Aschauer H, Aszòdi A, Stuetz A. Percutaneous absorption of drugs used in atopic eczema: pimecrolimus permeates less through skin than CS and tacrolimus. Int J Pharmacol 2004; 269: 29-35.

10 Leung DY, Bieber T. Atopic dermatitis. Lancet 2003; 361: 151-60.

11 Girolomoni G, Abeni D, Masini C et al. The epidemiology of atopic dermatitis in Italian schoolchildren. Allergy 2003; 58: 420-5. 
12 Fivenson D, Arnold RJ, Kaniecki DJ et al. The effect of atopic dermatitis on total burden of illness and quality of life on adults and children in a large managed care organization. J Manag Care Pharm 2002; 8: 333-42.

13 Ellis CN, Drake LA, Prendergast MM et al. Cost of atopic dermatitis and eczema in the United States. J Am Acad Dermatol 2002; 46: 361-70.

14 Eichenfield LF, Lucky AW, Boguniewicz M et al. Safety and efficacy of pimecrolimus (ASM 981) cream $1 \%$ in the treatment of mild and moderate atopic dermatitis in children and adolescents. J Am Acad Dermatol 2002; 46: 495-504.

15 Eichenfield LF, Lucky AW, Langley RG et al. Use of pimecrolimus cream 1\% (Elidel) in the treatment of atopic dermatitis in infants and children: the effects of ethnic origin and baseline disease severity on treatment outcome. Int J Dermatol 2005; 44: $70-5$.

16 Leo HL, Bender BG, Leung SB et al. Effect of pimecrolimus cream $1 \%$ on skin condition and sleep disturbance in children with atopic dermatitis. J Allergy Clin Immunol 2004; 114: 691-3.

17 Kempers S, Boguniewicz M, Carter E et al. A randomized investigator-blinded study comparing pimecrolimus cream $1 \%$ with tacrolimus ointment $0.03 \%$ in the treatment of pediatric patients with moderate atopic dermatitis. J Am Acad Dermatol 2004; 51: 515-25.

18 Kapp A, Papp K, Bingham A et al. Long-term management of atopic dermatitis in infants with topical pimecrolimus, a nonsteroid anti-inflammatory drug. J Allergy Clin Immunol 2002; 110: 277-84.

19 Papp KA, Werfel T, Folster-Holst R et al. Long-term control of atopic dermatitis with pimecrolimus cream $1 \%$ in infants and young children: a two-year study. J Am Acad Dermatol 2005; 52: 240-6.

20 Meurer M, Fartasch M, Albrecht G et al. Long-term efficacy and safety of pimecrolimus cream $1 \%$ in adults with moderate atopic dermatitis. Dermatology 2004; 208: 365-72.

21 Luger TA, Lahfa M, Folster-Holst R et al. Long-term safety and tolerability of pimecrolimus cream $1 \%$ and topical corticosteroids in adults with moderate to severe atopic dermatitis. J Dermatol Treat 2004; 15: 169-78.

22 Meingassner JG, Grassberger M, Fahrngruber $\mathrm{H}$ et al. A novel anti-inflammatory drug, SDZ ASM 981, for the topical and oral treatment of skin diseases: in vivo pharmacology. $\mathrm{Br} J$ Dermatol 1997; 137: 568-76.

23 Wahn U, Bos JD, Goodfield M et al. Efficacy and safety of pimecrolimus cream in the long-term management of atopic dermatitis in children. Pediatrics 2002; 110 (1 Part 1): e2.
24 Papp KA, Breuer K, Meurer $M$ et al. Long-term treatment of atopic dermatitis with pimecrolimus cream $1 \%$ in infants does not interfere with the development of protective antibodies after vaccination. J Am Acad Dermatol 2005; 52: 247-53.

25 Gribetz C, Ling M, Lebwohl M et al. Pimecrolimus cream 1\% in the treatment of intertriginous psoriasis: a double-blind, randomized study. J Am Acad Dermatol 2004; 51: 731-8.

26 Rigopoulos D, Ioannides D, Kalogeromitros D et al. Pimecrolimus cream $1 \%$ vs. betamethasone 17 -valerate $0.1 \%$ cream in the treatment of seborrhoeic dermatitis. A randomized open-label clinical trial. Br J Dermatol 2004; 151: 1071-5.

27 Belsito DV, Fowler JF Jr, Marks JG Jr et al. Pimecrolimus cream 1\%: a potential new treatment for chronic hand dermatitis. Cutis 2004; 73: 31-8.

28 Esquivel-Pedraza L, Fernandez-Cuevas L, Ortiz-Pedroza G et al. Treatment of oral lichen planus with topical pimecrolimus $1 \%$ cream. Br J Dermatol 2004; 150: 771-3.

29 Dissemond J, Schroter S, Franckson T et al. Pimecrolimus in an adhesive ointment as a new treatment option for oral lichen planus. Br J Dermatol 2004; 150: 782-4.

30 Kreuter A, Gambichler T, Breuckmann F et al. Pimecrolimus $1 \%$ cream for cutaneous lupus erythematosus. I Am Acad Dermatol 2004; 51: 407-10.

31 Lim SJ, Love EW. Steroid-free pimecrolimus (Elidel) for monotherapy of lichen planus. J Drugs Dermatol 2004; 3: 563-4.

32 Mayoral FA, Gonzalez C, Shah NS, Arciniegas C. Repigmentation of vitiligo with pimecrolimus cream: a case report. Dermatology 2003; 207: 322-3.

33 Ziemer M, Gruhn B, Thiele JJ, Elsner P. Treatment of extensive chronic cutaneous graft-versus-host disease in an infant with topical pimecrolimus. J Am Acad Dermatol 2004; 50: 946-8.

34 Mrowietz U, Wustlich S, Hoexter G et al. An experimental ointment formulation of pimecrolimus is effective in psoriasis without occlusion. Acta Derm Venereol 2003; 83: 351-3.

35 Thaci D, Steinmeyer K, Ebelin ME et al. Occlusive treatment of chronic hand dermatitis with pimecrolimus cream $1 \%$ results in low systemic exposure, is well tolerated, safe, and effective. An open study. Dermatology 2003; 207: 37-42.

36 Rappersberger K, Komar M, Ebelin ME et al. Pimecrolimus identifies a common genomic anti-inflammatory profile, is clinically highly effective in psoriasis and is well tolerated. J Invest Dermatol 2002; 119: 876-87.

37 Harbour R, Miller J. A new system for grading recommendations in evidence based guidelines. BMJ 2001; 323: 334-6.

Paper received March 2005, accepted April 2005 